\section{RELATO DE EXPERIÊNCIA DO ENSINO DE SAÚDE PÚBLICA POR MEIO DE DISCIPLINA CONDENSADA NA GRADUAÇÃO EM FISIOTERAPIA}

\author{
EXPERIENCE REPORT ON PUBLIC HEALTH TEACHING \\ THROUGH CONDENSED SUBJECT IN PHYSIOTHERAPY \\ UNDERGRADUATE SCHOOL
}

Autor Correspondente

Alberto Sumiya

E-mail: a.sumiya@ufsc.br

${ }^{1}$ Coordenadoria Especial de Biociências e Saúde Única, Universidade Federal de Santa Catarina

\begin{abstract}
RESUMO
Contextualização: o ensino de saúde pública nos cursos de graduação em fisioterapia tem se consolidado como um compromisso com o Sistema Único de Saúde (SUS) brasileiro. Nesse sentindo, os seus currículos se esforçam para se tornar adaptados às necessidades de saúde e informados sobre possibilidades como a disciplina em formato condensado. Descrição da experiência: um relato de experiência sobre o ensino de saúde pública em formato condensado na graduação em fisioterapia, a qual foi desenvolvida em cinco semanas com três encontros semanais, entre aulas práticas e teóricas. Ao final da disciplina, foi aplicado um questionário com 12 perguntas, sendo 10 do tipo Likert e 2 abertas. Impactos: os estudantes apresentaram aquisição de conhecimento quando se comparou o antes e o depois, e mostraram-se satisfeitos com a disciplina condensada. Considerações finais: perante os impactos, acredita-se que disciplinas no formato condensado possuem potencial para serem desenvolvidas na graduação em fisioterapia. No que concerne a este relato, verificou-se que as aulas práticas precisavam ser mais bem dimensionadas para suprir as necessidades apontadas pelos estudantes. Por fim, sugerem-se mais discussões colegiadas sobre a temática, além de mais estudos exploratórios que visem contrastar fases, disciplinas, cargas horárias, conteúdos, momento de realização e visão institucional, que seriam mais adequados a esse formato.
\end{abstract}

Palavras-chave: Fisioterapia; Saúde pública; Aprendizagem; Educação.

\begin{abstract}
Contextualization: the teaching of public health in undergraduate courses in physiotherapy has been consolidated as a commitment to the Brazilian Unified Health System (SUS). In this sense, their curricula strive to become adapted to health needs and informed about possibilities such as the subject in a condensed format. Description of the experience: an experience report on the teaching of public health in a condensed format in the undergraduate course in physiotherapy, which was developed in five weeks with three weekly meetings, between practical and theoretical classes. At the end of the course, a questionnaire with 12 questions was applied, 10 of which were Likert and 2 open. Impacts: students showed knowledge acquisition when comparing before and after and were satisfied with the condensed subject. Final considerations: in view of the impacts, it is believed that subjects in the condensed format have the potential to be developed in undergraduate physiotherapy. Regarding this report, it was found that the practical classes needed to be better sized to meet the needs pointed out by the students. Finally, more collegiate discussions on the theme are suggested, in addition to more exploratory studies aimed at contrasting phases, disciplines, workloads, content, time of realization and institutional vision, which would be more appropriate to this format.
\end{abstract}

Keywords: Physical therapy specialty; Public health; Learning; Education. 


\section{CONTEXTUALIZAÇÃO}

Em 2018, iniciaram-se as discussões sobre a proposta de mudança curricular do curso de graduação em fisioterapia da Universidade Federal do Amazonas (Ufam), na cidade de Coari, considerada a segunda maior do estado, na medida em que o Projeto Político-Pedagógico (PPP) e a matriz curricular não tinham sofrido alterações desde a criação.

$\mathrm{O}$ intuito era atualizar os planos de ensino, alterando desde a denominação das disciplinas, ementas e cargas horárias até o remanejamento de alocação, além de eliminar e/ou criar outras. Nesse processo, aventou-se a possibilidade de operacionalizar as disciplinas condensadas, o que, em tese, ajudaria a liberar tempo para os docentes trabalharem com projetos de ensino, pesquisa e extensão, para se conectarem a outros centros educacionais e superar o extremo isolamento geográfico e as limitações tecnológicas.

Uma disciplina condensada primordialmente - porém não somente - ganha tempo e promove imersão no conteúdo com maximização do aprendizado, que, quando estruturado adequadamente, produz as habilidades esperadas. Considera-se aqui como disciplina condensada $^{1}$ aquela que é operacionalizada em menor espaço de tempo, geralmente menos da metade das semanas de um semestre letivo regular, contudo mantendo a carga horária. Em termos de esclarecimento, na literatura, observam-se termos variados para essa perspectiva, como, por exemplo, disciplina comprimida $^{2}$ e disciplina abreviada ${ }^{3}$, e de maneira correlata ainda aparecem ensino blocado ${ }^{4}$ e ensino intensivo ${ }^{5}$.

A partir desse contexto, a disciplina de Saúde Pública (dSP) foi lecionada condensadamente. Levantou-se a hipótese de que a configuração despertaria maior interesse dos estudantes por esse campo em contraste com outras especialidades consideradas aparentemente mais atraentes devido às práticas manuais e utilização de tecnologias eletrônicas, mantendo a qualidade e um bom nível da aquisição de conhecimento. Considerou -se, ainda, que a experiência permitira apontar vantagens e limitações para futuros movimentos da mudança curricular; e, por fim, observou-se também a inexistência de literatura sobre o tema, tendo como objeto de estudo a própria fisioterapia.

Dessa forma, o objetivo deste artigo foi descrever o desenvolvimento de uma disciplina condensada, apresentando os conteúdos abordados e as estratégias didáticas utilizadas, para discutir a influência do tempo de duração da disciplina sobre os níveis de conhecimento e a satisfação dos estudantes.

\section{CONSTRUINDO A PESQUISA}

Este é relato de experiência sobre a condensação da dSP do curso de fisioterapia da Ufam, no Instituto de Biociências e Saúde (ISB), do Campus de Coari, no primeiro semestre de 2019, e que contou com três encontros semanais com previsão de duração de quatro horas entre teoria e prática, durante cinco semanas consecutivas.

A dSP é semestral (60 horas), envolvendo aulas teóricas e práticas, desenvolvida regularmente em 15 semanas, sendo o seu pré-requisito a disciplina de Epidemiologia Geral (60 horas). No seu eixo de conhecimento, encontram-se a disciplina Determinantes Sócio-Econômicos-Antropológicos da Saúde (30 horas) e o Estágio Supervisionado em Saúde Pública (150 horas).

A ementa da dSP, conforme o PPP, inclui: saúde pública brasileira - aspectos históricos, práticos e processuais; aspectos gerais e especiais de higiene e saúde pública no país; o papel do profissional de saúde da área de fisioterapia; legislação do Sistema Único de Saúde (SUS). Seu objetivo geral, de acordo com o plano de ensino, é conhecer o aporte teórico necessário para a prática assistencial em saúde pública, no que se refere aos aspectos do indivíduo, da família e da comunidade em termos de epidemiologia, oportunizando refletir sobre a problemática que envolve 
as políticas de saúde pública e a contribuição do fisioterapeuta na realidade social e na implementação de práticas de saúde que contribuam para a melhoria da qualidade da saúde pública.

Como parte da experiência de ensino condensado, o professor buscou incrementar o feedback aplicando um questionário de 12 perguntas (Tabela 1) ao fim da disciplina. As primeiras 10 perguntas eram do tipo Likert com escore variando de 1 a 5 para assinalar, sem proposição de intervalos de categorização, interpretando-se como melhor resultado escores mais altos.
As duas últimas perguntas eram abertas e foram analisadas pelo NVIVO Software Versão 11 da QSR Internacional, com parâmetros: frequência das primeiras 50 palavras, comprimento mínimo de 5 caracteres e o agrupamento de palavras derivadas. Por último, somente foram analisados os questionários totalmente preenchidos, sem nenhuma reposta em branco e sem rasuras.

Tabela 1. Questionário de autoavaliação e contribuição (Brasil, 2019)

\begin{tabular}{|c|c|c|c|c|c|c|}
\hline $\mathbf{N}^{\circ}$ & Pergunta & Ruim & Razoável & Bom/Boa & $\begin{array}{l}\text { Muito } \\
\text { bom/boa }\end{array}$ & Excelente \\
\hline 01 & $\begin{array}{l}\text { O meu conhecimento anterior da } \\
\text { disciplina de saúde coletiva }\end{array}$ & 1 & 2 & 3 & 4 & 5 \\
\hline 02 & $\begin{array}{l}\text { O meu conhecimento sobre a ementa } \\
\text { e o objetivo da disciplina }\end{array}$ & 1 & 2 & 3 & 4 & 5 \\
\hline 03 & $\begin{array}{l}\text { O meu grau de motivação inicial com a } \\
\text { disciplina }\end{array}$ & 1 & 2 & 3 & 4 & 5 \\
\hline 04 & A minha dedicação para a disciplina & 1 & 2 & 3 & 4 & 5 \\
\hline 05 & A minha participação nas aulas & 1 & 2 & 3 & 4 & 5 \\
\hline 06 & $\begin{array}{l}\text { A minha motivação ao final da } \\
\text { disciplina }\end{array}$ & 1 & 2 & 3 & 4 & 5 \\
\hline 07 & $\begin{array}{l}\text { O meu conhecimento atual sobre a } \\
\text { disciplina }\end{array}$ & 1 & 2 & 3 & 4 & 5 \\
\hline 08 & $\begin{array}{l}\text { A metodologia de ensino utilizada na } \\
\text { disciplina }\end{array}$ & 1 & 2 & 3 & 4 & 5 \\
\hline 09 & $\begin{array}{l}\text { A metodologia de avaliação utilizada } \\
\text { na disciplina }\end{array}$ & 1 & 2 & 3 & 4 & 5 \\
\hline 10 & $\begin{array}{l}\text { O meu nível de satisfação com a } \\
\text { disciplina }\end{array}$ & 1 & 2 & 3 & 4 & 5 \\
\hline 11 & \multicolumn{6}{|c|}{$\begin{array}{l}\text { Na sua opinião, qual a importância do fisioterapeuta e/ou da fisioterapia na atenção primária à } \\
\text { saúde? }\end{array}$} \\
\hline 12 & \multicolumn{6}{|c|}{ Você teria alguma sugestão para a disciplina? } \\
\hline
\end{tabular}

Fonte: elaboração própria (2019). 


\section{A DESCRIÇÃO DA EXPERIÊNCIA}

Antes de tudo, é importante informar que não foi intenção deste relato realizar uma descrição excessivamente densa e exaustiva, mas oferecer os contornos gerais do seu desenvolvimento, tendo em vista a própria modalidade metodológica e a restrição de espaço na revista.

Na primeira aula, diferenciou-se saúde pública de saúde coletiva, evidenciando a importância de ambas em benefício da saúde e desenvolvimento social. No mesmo momento, problematizaram-se o processo saúde-doença e os Determinantes Sociais em Saúde (DSS). O encontro seguinte aconteceu em uma Unidade Básica de Saúde (UBS) localizada em uma das regiões consideradas mais pobres e com alto nível de violência. Contudo, apresenta uma das melhores infraestruturas da cidade, inclusive um espaço próprio para a fisioterapia.

A ideia de poder enxergar, interagir, sentir e analisar a UBS como espelho de um sistema maior encaminhou para a história do SUS, seus princípios e fundamentos. Naturalmente, emergiram concatenados os modelos e os níveis de atenção, sempre ressaltando-se a centralidade da Atenção Primária à Saúde (APS) como porta de entrada, que fornece sentido ordenador dentro da rede de atenção. Acrescentaram-se ao debate indicadores sociais, econômicos e de saúde da população brasileira em referência aos DSS.

Imediatamente, criou-se uma ponte para adentrar nos temas relacionados com Estratégia Saúde da Família (ESF), Equipe Saúde da Família (EqSF), Núcleo Ampliado de Saúde da Família (Nasf), Agente Comunitário de Saúde (ACS) e a relação destes com a fisioterapia. Além disso, indagou-se sobre a luta política do fisioterapeuta para se engajar no sistema público de saúde e na ESF e a necessidade de conscientização sobre a integralidade da saúde. A provocação fez surgir a discussão sobre tecnologias leves e a humanização como forma de produção de vínculos com corpos, afetos e itinerários terapêuticos, que a técnica isoladamente não dá conta de solucionar.
Convidou-se, em seguida, uma egressa do curso que trabalhava no Nasf para compartilhar a sua experiência em uma roda de conversa. Ela discorreu sobre os obstáculos diários de andarilhar sob o calor ardente do Amazonas, da receptividade das famílias e/ou das recusas em aceitar a sua presença, dos enfrentamentos com a gestão municipal para incrementar o serviço e do receio da instabilidade empregatícia, da descontinuidade devido às trocas de mandatos políticos. Assim, emanaram-se os conceitos e as práticas de promoção, prevenção e educação, que ajudaram a desmistificar o estigma curativo da profissão que ainda sobrevoa a formação.

Por conseguinte, introduziu-se a territorialização e como procedê-la, tentando fazer o estudante ultrapassar, e ao mesmo tempo atrelar, a questão física-material do ambiente, para captar as conjunturas que determinam as vulnerabilidades e a vigilância em saúde. Dando continuidade, foram trazidos instrumentos avaliativos: o Apgar Familiar, Genograma Familiar e Ecomapa; como qualificação do trabalho, propôs-se uma atividade na qual cada estudante deveria realizar o seu genograma familiar olhando para os aspectos da saúde. A trajetória culminou com apresentações e discussão de políticas públicas de saúde, buscando refletir sobre a relação com a fisioterapia.

Além disso, duas outras rodas de conversa foram realizadas: a primeira sobre a Política Nacional de Alimentação e Nutrição com uma docente do curso de nutrição da Ufam; e a segunda sobre saúde mental com a psicóloga da Ufam/ ISB. Constatou-se, a despeito da floresta e dos rios, que a cidade tem hábitos alimentares modernos com consumo de produtos processados ou ultraprocessados, determinando aumento da incidência de várias doenças crônicas. No âmbito da saúde mental, a discussão gerou apontamentos sobre a carência de medidas preventivas precoces e das dificuldades enfrentadas pela falta de serviços especializados na cidade, como médico psiquiatra e clínica de psicologia.

No geral, a expectativa do professor da disciplina era que os conteúdos fossem 
se entrelaçando e, assim, favorecesse a percepção, pelo estudante, de coerência entre os assuntos e de coesão para a prática (Quadro 1).

Quadro 1. Sumário dos conteúdos trabalhados na dSP (Brasil, 2019)

\begin{tabular}{|l|l|}
\hline Conteúdo (s) & \multicolumn{1}{c|}{ Metodologia } \\
\hline $\begin{array}{l}\text { Diferença entre saúde pública e saúde coletiva } \\
\text { Processo saúde-doença e DSS }\end{array}$ & $\begin{array}{l}\text { Aula expositiva dialogada, estudo } \\
\text { dirigido e vídeos }\end{array}$ \\
\hline $\begin{array}{l}\text { SUS, modelos e níveis de atenção } \\
\text { ESF, EgSF, Naşf e ACS }\end{array}$ & $\begin{array}{l}\text { Aula expositiva dialogada, visitas à } \\
\text { UBS e relatórios }\end{array}$ \\
\hline $\begin{array}{l}\text { Promoção, prevenção e educação em saúde } \\
\text { Papel do fisioterapeuta na APS }\end{array}$ & $\begin{array}{l}\text { Roda de conversa, visitas domiciliares } \\
\text { e produção de material informativo }\end{array}$ \\
\hline Instrumentos de avaliação na APS & $\begin{array}{l}\text { Aula expositiva dialogada, resolução } \\
\text { de problemas e estudo de casos }\end{array}$ \\
\hline Políticas Públicas de Saúde no Brasil & $\begin{array}{l}\text { Aula expositiva dialogada, rodas de } \\
\text { conversa, seminários e filmes }\end{array}$ \\
\hline Doenças Crónicas Não Transmissíveis (DCNT) & \begin{tabular}{l} 
Aula expositiva dialogada e debates \\
\hline
\end{tabular} \\
\hline
\end{tabular}

Fonte: elaboração própria (2019). 


\section{IMPACTOS}

Participaram da dSP 49 estudantes; 41 responderam ao questionário, mas 8 foram excluídos por rasuras e/ou dificuldade na identificação da resposta correta, assim, permaneceram válidos para análise 33 questionários. Não houve avaliação com provas, porém, atribuíram-se notas por meio da participação nos exercícios, nas atividades, nos trabalhos práticos e nas visitas a UBS e domiciliares. A nota média da turma foi de $8.05 \pm 0.61$, mas houve três reprovações por faltas.

Assim sendo, ao se comparar as perguntas 1 e 7, demonstrou-se descritivamente que houve aquisição de conhecimento (Gráfico 1), inclusive observável por meio dos números absolutos na legenda. Em outras palavras, a maioria dos estudantes percebiam seu nível de conhecimento como "bom" inicialmente; e, ao término da disciplina, passaram a enxergá-lo como "muito bom" (39,3\%) $(n=13)$ e excelente $(36,3 \%)(n=12)$.

Gráfico 1. Nível de conhecimento sobre saúde pública antes e depois (Brasil, 2019)

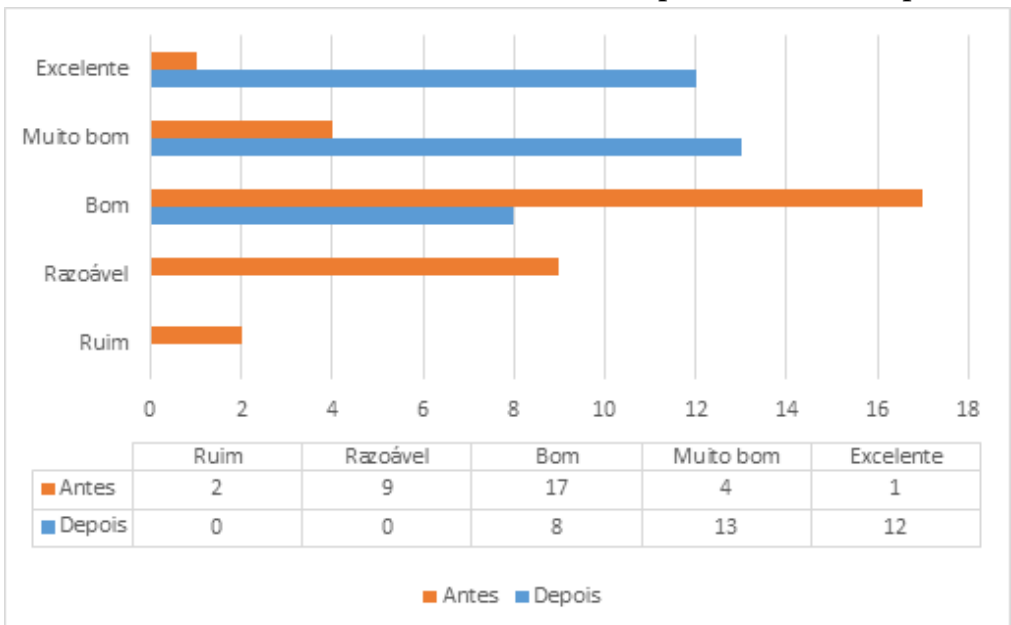

Fonte: elaboração própria (2019). 
Percebeu-se também que a aquisição de conhecimento em saúde pública aparentemente refletiu no resultado sobre nível de satisfação final com a dSP, em que a maioria $(n=25)(75,7 \%)$ avaliou com escore máximo, e apenas um estudante avaliou como razoável, conforme também indicam os números absolutos na legenda (Gráfico 2).

Gráfico 2. Nível de satisfação após o término da dSP (Brasil, 2019)

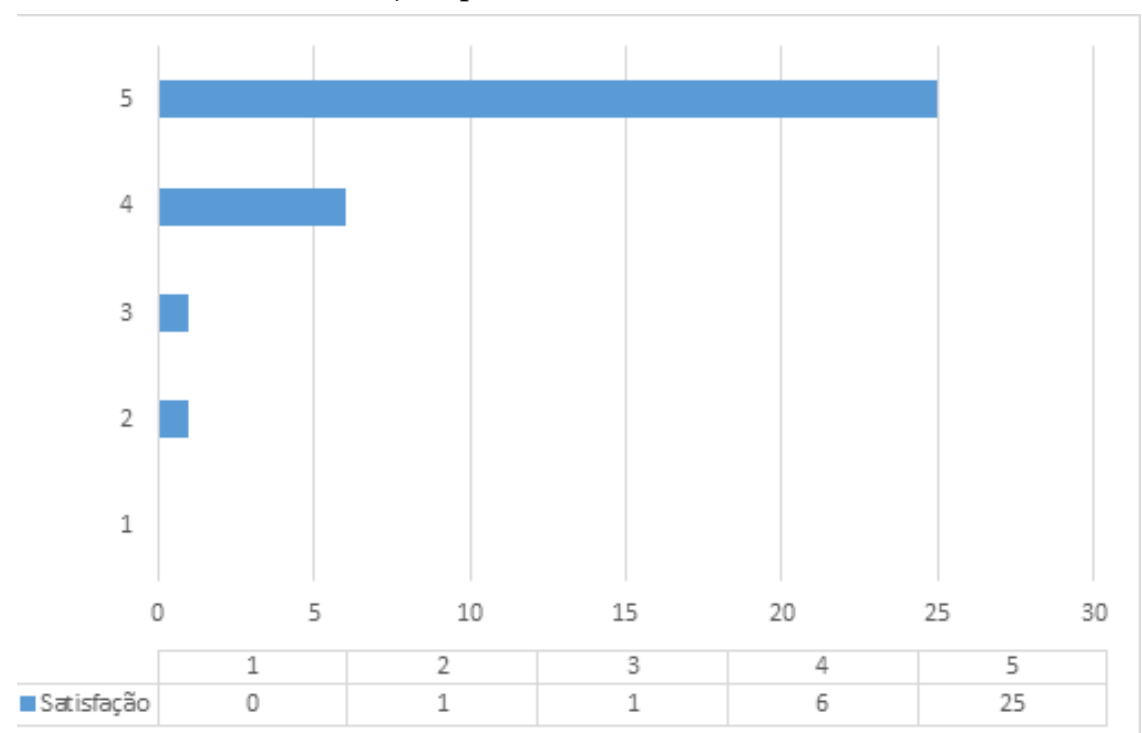

Fonte: elaboração própria (2019).

Como representação dos discursos das perguntas 11 e 12, extraíram-se trechos mais significativos, que foram agregados e colocados ao lado da sua respectiva nuvem de palavra (Quadro 2). Embora seja perceptível a impregnação conceitual dada pela dSP, interpretou-se que os termos voltados ao passado reabilitador ainda sobrevoam as falas. As sugestões foram quase unanimemente direcionadas à solicitação de mais aulas práticas na UBS e nas comunidades. Como críticas, apontaram-se que a disciplina foi cansativa, que preferia o modelo regular e que gostaria de mais explicações sobre o trabalho do fisioterapeuta na APS.

Assim sendo, os resultados encontrados neste relato de experiência mostram que os estudantes tiveram aquisição de conhecimento e apresentaram-se satisfeitos ao final da dSP no formato condensado. De acordo com Scyoc e Gleason ${ }^{6}$, o tempo é uma condição necessária para o aprendizado e para a aquisição de conhecimento, podendo ser utilizado em vários níveis de intensidade/duração.

Austin e Gustafson ${ }^{7}$ fizeram um levantamento retrospectivo do desempenho acadêmico de 45 mil estudantes e verificaram que disciplinas intensivas resultaram em notas maiores comparado aos sujeitos que frequentaram disciplinas regulares de 16 semanas. De forma semelhante, Sheldon e Durdella ${ }^{2}$ analisaram 21.165 registros de três cursos de graduação, dos quais 3.360 eram de estudantes que haviam se matriculado em disciplinas comprimidas, no formato de 6-8 semanas. O sucesso em completar a disciplina foi major entre os que cursaram disciplinas comprimidas de 8 semanas. Willianson ${ }^{8}$ também encontrou que estudantes que cursaram uma disciplina denominada de minissemestre (comprimida), constituída de 10 dias, tiveram melhor realização nas atividades de laboratório e leitura de problemas do que aqueles que cursaram as 16 semanas 
normais. Assim, para alguns estudantes, as disciplinas condensadas são mais efetivas $^{1}$ e, pelo menos, tão exigente quanto as disciplinas regulares ${ }^{3}$, em que os estudantes costumam pontuar melhor ${ }^{6}$.

Quadro 2. Trechos de discursos dos estudantes e nuvens de palavras (Brasil, 2019)

\begin{tabular}{|c|c|}
\hline Discurso & Nuvem \\
\hline $\begin{array}{l}{[\ldots] \text { o fisioterapeuta trabalha na prevenção, }} \\
\text { promoção e reabilitação do indivíduo, sendo visto } \\
\text { não apenas como reabilitador e sim participante do } \\
\text { conjunto de ações desenvolvidas para os } \\
\text { pacientes/usuários do SUS. [...] é fundamental na } \\
\text { atenção primária para o diagnóstico precoce e } \\
\text { orientação na comunidade. [...] atuação na } \\
\text { promoção e prevenção a partir de práticas } \\
\text { saudáveis de modo individual e coletiva, a fim de } \\
\text { evitar gastos futuros com prevenção secundária e } \\
\text { terciária. }\end{array}$ & 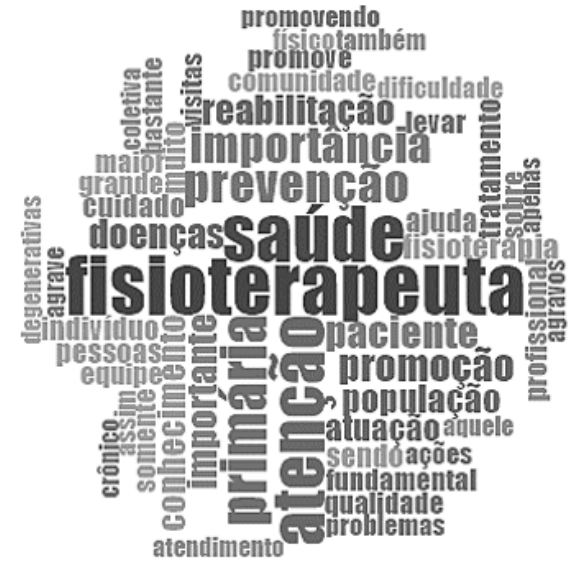 \\
\hline $\begin{array}{l}\text { Sugestão de levar mais vezes os alunos a } \\
\text { acompanharem os estagiários de fisioterapia para } \\
\text { assim ver e aprender com as práticas sobre a } \\
\text { atuação do fisioterapeuta principalmente nos } \\
\text { estágios que nos mostra uma realidade daquilo que } \\
\text { iremos ter e passar futuramente. }\end{array}$ & 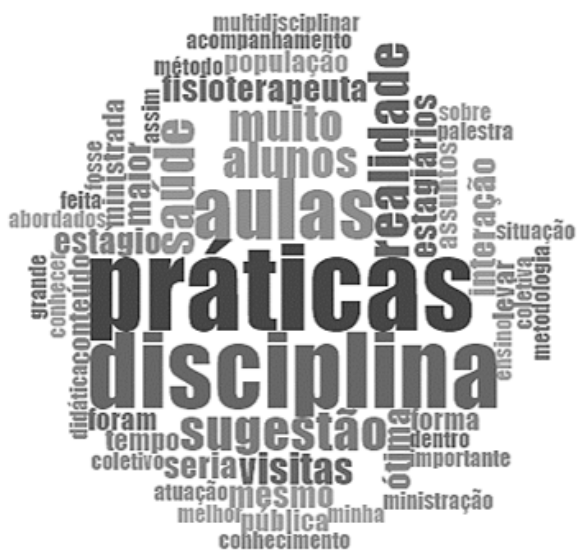 \\
\hline
\end{tabular}

Fonte: elaboração própria (2019). 
De modo geral, os estudos citados anteriormente sugerem que os discentes aprendem mais e melhor em disciplinas condensadas ou que a diferença entre o formato condensado e regular é pequena. Daniel ${ }^{9}$ afirmou que, em sua pesquisa, os estudantes reportaram alto nível de motivação com percepção de maior sucesso devido à maior concentração $0^{3}$ nos resultados do aprendizado, participação em discussões e presença mais frequente nas aulas. A instituição, segundo o autor ${ }^{9}$, observou que houve mais discussões em profundidade e que as atividades eram mais experienciais, reduzindo então a possibilidade de desistências.

Esses achados são provavelmente devido à imersão e à falta de distrações interferindo ${ }^{8}$. Contudo, a crítica ao formato vem da defesa de que a retenção do conhecimento é maior quando o aprendizado é espaço $\mathrm{O}^{1,3}$, produzindo memória de longo prazo $^{8}$. Entretanto, para Anastasi ${ }^{3}$, essa é uma crença negativa infundada, que pode ser superada com medidas como ${ }^{1}$ : maximizar as oportunidades de prática e feedback para aumentar a retenção de longo prazo; produzir intencionalmente maneiras de reengajamento e reavaliação; subdividir as avaliações para que estas sejam mais frequentes (quizzes semanais).

Este relato de experiência proporcionou com potência muitos insights, que serviram para discutir o currículo nos cursos de fisioterapia. As limitações estão relacionadas com a descrição de uma experiência que foi isolada e que não se reproduziu novamente para poder explorar tempos de duração em semanas, métodos de ensino e avaliação, além da inteiração com as outras disciplinas lecionadas no mesmo período. A dSP com carga horária de 60 horas não consegue cobrir conteúdos, como, por exemplo, gestão em saúde (organização, planejamento, avaliação, financiamento), comunicação (literacy) em saúde e vigilância em saúde, entre outros; o que realmente denuncia a necessidade de mudança curricular no curso de graduação em fisioterapia da Ufam.

\section{CONSIDERAÇÕES FINAIS}

Sumiya
Perante os impactos, acredita-se que disciplinas no formato condensado possuem potencial para serem desenvolvidas na graduação em fisioterapia. No que concerne a este relato, verificou-se que as aulas práticas precisavam ser mais bem dimensionadas para suprir as necessidades apontadas pelos estudantes. Por fim, sugerem-se mais discussões colegiadas sobre a temática, além de mais estudos exploratórios que visem contrastar fases, disciplinas, cargas horárias, conteúdos, momento de realização e visão institucional, que seriam mais adequados a esse formato.

\section{REFERÊNCIAS}

1. Walsh KP, Sanders M, Gadgil S. Equivalent but not the Same: Teaching and Learning in Full Semester and Condensed Summer Courses. Coll Teach. 2019;67(2):138-49. doi:10.1080/875675 55.2019 .1579702

2. Sheldon CQ, Durdella NR. Success rates for students taking compressed and regular length developmental courses in the community college. Community Coll J Res Pract. 2010;34(1-2):39-54. doi:10.1080/10668920903385806

3. Anastasi JS. Full-Semester and Abbreviated Summer Courses: An Evaluation of Student Performance. Teach Psychol. 2007;34(1):19-22. doi:10.1080/00986280709336643

4. Dixon L, O'Gorman V. 'Block teaching'-exploring lecturers' perceptions of intensive modes of delivery in the context of undergraduate education. J Furth High Educ. 2020;44(5):583-95. doi:10.1 080/0309877X.2018.1564024

5. Harvey M, Power M, Wilson M. A review of intensive mode of delivery and science subjects in Australian universities. J Biol Educ. 2017;51(3):315-25. doi :10.1080/00219266.2016.1217912

6. Van Scyoc LJ, Gleason J. Traditional or intensive course lengths? a comparison of outcomes in economics learning. J Econ Educ. 1993;24(1):15-22. doi:10.1 080/00220485.1993.10844775 


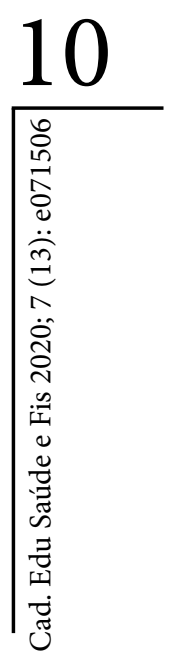

7. Austin AM, Gustafson L. Impact of Course Length on Student Learning. J Econ Financ Educ. 2006;5(1):26-37.

8. Williamson KC. The Effect of Course Time Compression on Academic Performance in Construction Surveying. Int J Constr Educ Res. 2018;14(4):277-94. doi :10.1080/15578771.2017.1365783

9. Daniel E. A Review of Time-Shortened Courses across Disciplines. Coll Stud J. 2000;34(2):298.

Recebido: 24/02/2020

Aprovado: 21/09/2020 